\title{
Multiple sclerosing haemangiomas of the lung
}

\author{
K. JoSHI \\ M.D.* \\ S. K. ShankaR \\ M.D.* \\ N. Gopinath \\ M.S., M.S. $\dagger$ \\ RAMESH KUMAR \\ M.D. $\ddagger$ \\ P. CHOPRA \\ M.D.* \\ Departments of Pathology*, Cardiothoracic Surgery $\dagger$, and Medicine $\ddagger$,
All India Institute of Medical Sciences, New Delhi-110016 India
}

\begin{abstract}
Summary
A case of multiple sclerosing haemangiomas of the lung is described from a 40-year-old woman, who presented with haemoptysis. A chest X-ray revealed multiple circumscribed coin lesions in both lungs. A right upper lobectomy was done for diagnosis. The patient has remained well for 2 years after surgery. Multiplicity of tumour masses of pulmonary sclerosing haemangiomas is extremely rare and, although benign, may pose a great diagnostic problem.
\end{abstract}

\section{Introduction}

Sclerosing haemangioma of the lung was first described by Liebow and Hubell (1956). These lesions have been reported under different names, namely xanthoma, fibroxanthoma, plasma cell granuloma, histiocytoma and alveolar angioblastoma (Arean and Wheat, 1962; Bahadori and Liebow, 1973; Gross and Wolbach, 1943; Hill and Eggleston, 1972; Lago et al., 1976). Characteristically there are minimal clinical symptoms other than haemoptysis and the lesions are generally detected on routine X-ray as round, well circumscribed shadows, generally single, of a slow rate of growth In the experience of J. T. Gmelich and A. A. Liebow (personal communication, 1977) only 4 of the 75 cases of sclerosing haemangiomas were multifocal. Another case of this rare entity with numerous tumour nodules is now presented.

\section{Case report}

A 40-year-old woman came to the All India Institute of Medical Sciences Hospital because of frequent haemoptysis, minimal cough and breathlessness of one year's duration. Before this illness

Correspondence: Dr Kusum Joshi, M.D., Lecturer, Department of Pathology, Medical College, Rohtak-124001 (Haryana), India. she had been well. Physical examination revealed no abnormality. Routine haemogram and biochemical investigations were within normal limits. Repeated examination of the sputum showed no acid fast bacilli or malignant cells. A chest X-ray showed multiple circumscribed radio-opaque lesions in both lung fields.

Based mainly on the radiological findings, a clinical impression of malignant lesions in the lung was made and exploratory right thoracotomy was done. A firm mass was palpable in the posterior segment of the right upper lobe. Multiple smaller nodules were also felt in the right middle and lower lobes. A right upper lobectomy was performed for diagnosis of the condition.

The postoperative period was uneventful. The patient has remained asymptomatic for approximately 2 years after surgery.

\section{Pathology \\ Gross}

The right upper lobectomy specimen showed multiple tumour nodules, embedded in the pulmonary parenchyma. The largest tumour nodule measured $5 \times 4 \times 4 \mathrm{~cm}$ (Fig. 1). It was well encapsulated, but the capsule appeared to be continuous with the mucosa of the lobar bronchus. The cut surface of the tumour was greyish-white, fleshy, lobulated and was friable. Another well circumscribed tumour nodule, one $\mathrm{cm}$ in diameter was seen in the subpleural location. On sectioning, this appeared greyish-white, homogeneous and of a rubbery consistency. In addition, there were multiple small tumour nodules varying from 0.2 to $0.4 \mathrm{~cm}$ in diameter.

\section{Histology}

Microscopic examination of the tumour nodules revealed well circumscribed lesions compressing the 
surrounding pulmonary parenchyma. The tumour nodules were highly cellular, consisting of spindleshaped cells aligned in various directions with no particular pattern. These spindle cells were monomorphic, had ovoid to elongated nuclei and a scant cytoplasm. Intermingled with the tumour cells, there were many vascular spaces, varying from small capillaries to large vessels. In many areas, the tumour had a vasoformative appearance which was better appreciated in reticulin-stained tissue (Figs 2 and 3). Hyalinization of the vessel walls was seen prominently in the small tumour nodule. There were local aggregates of histiocytes, and infiltration of the stroma by inflammatory cells. There were no mitoses or giant cell formation. At the periphery, the lesion was growing into the interalveolar septa and protruded into the alveolar spaces, thus producing 'fissures' or 'papillary structures'. The alveolar lining in these areas was markedly proliferated and cuboidal.

\section{Discussion}

Angiomatous lesions of the lung have broadly been classified as (1) arteriovenous fistulas and (2) solid spheric haemangiomas by Mori (1968).

Liebow and Hubell (1956) described haemangiomatous lung tumours which they called sclerosing haemangioma. The histological features characteristic of this lesion, as they described it, are: an angiomatous lesion of a papillary nature; presence of

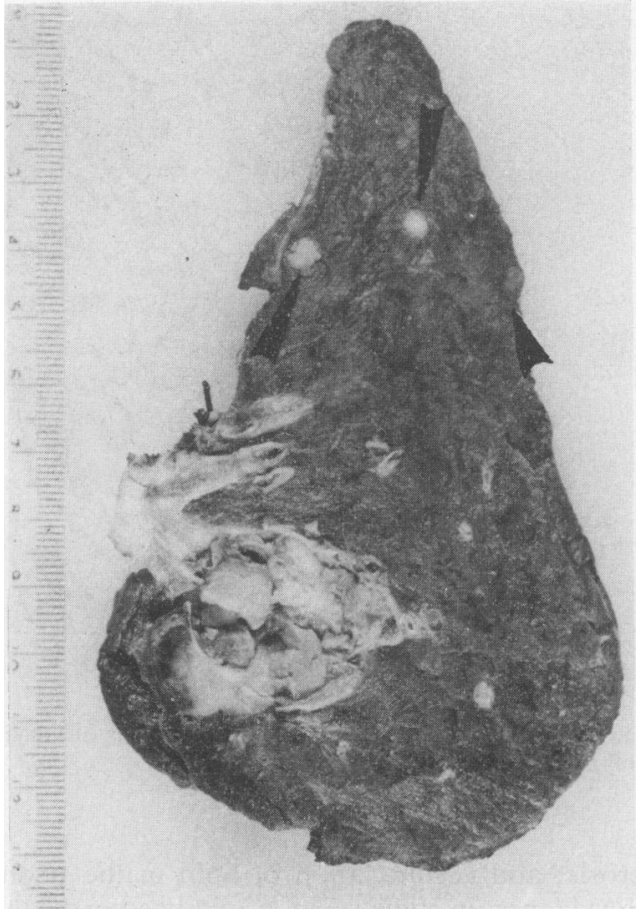

FIG. 1. Lobectomy specimen of the lung showing the main tumour mass in continuity with the major bronchus. The peripheral tumour nodules are indicated by arrows.

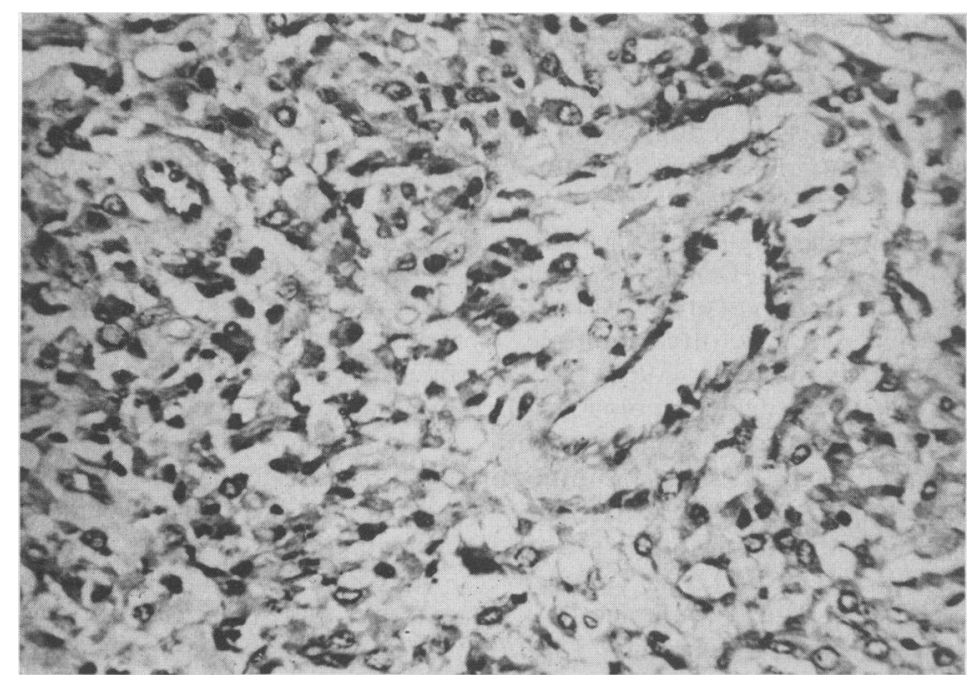

FIG. 2. Photomicrograph showing round to spindle shaped tumour cells with moderate degree of pleomorphism and formation of multiple vascular channels of varying sizes $(\mathrm{HE}, \times 180)$. 


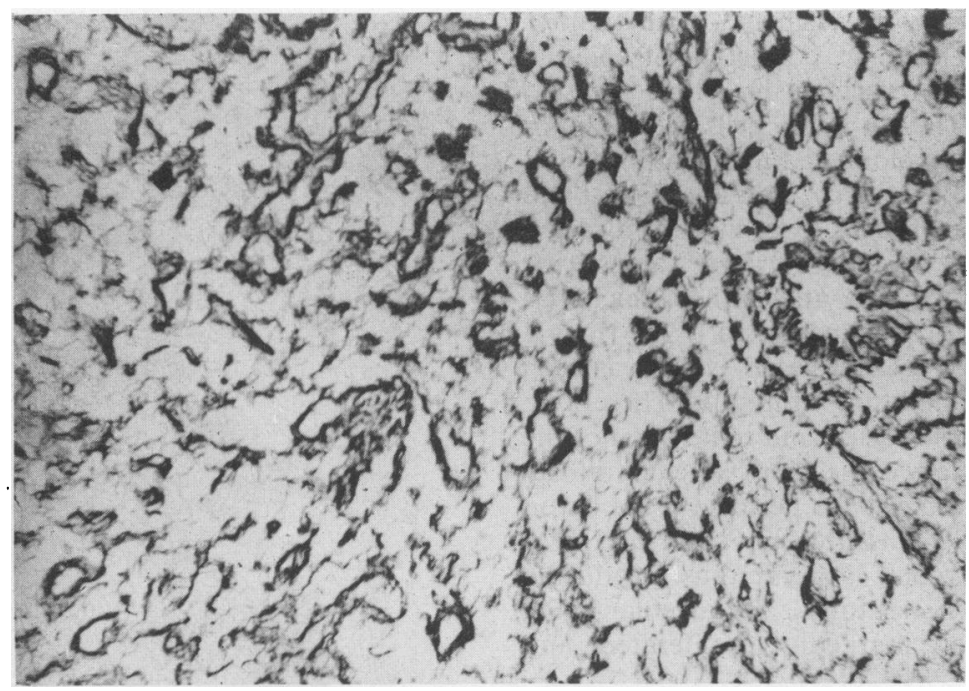

Fig. 3. Multiple thin walled vascular channels of varying sizes indicating the vasoformative nature of the lesion (Gordon and Sweet's Reticulin Stain, $\times 180$ ).

sclerosis; and accumulation of lipid in the stroma, with evidence, in some cases, of old haemorrhage.

In a review of 35 cases by Arean and Wheat (1962) $63 \%$ of the tumours occurred in females and mostly in individuals aged 30 to 40 years, although the age at the time of presentation varied from 3 months to 59 years. Many of the cases were clinically silent and were discovered at routine chest X-ray. Haemoptysis was noted in 23 out of $\mathbf{3 5}$ cases and its severity varied from minimal to massive. Cough, with or without production of sputum was the next common clinical sign, and there was often a history of recent pulmonary infection. In the present case the patient also presented with haemoptysis and cough.

Chest X-rays show these tumours as well circumscribed coin lesions and they therefore form an important differential diagnosis of coin lesions of the lung (Rubin, Rubin and Sicklick, 1958). Although benign angiomatous lesions elsewhere are considered to be developmental defects and are often multiple, these tumours are usually single (Mori, 1968). In a series of 10 cases of sclerosing haemangioma presenting as coin lesions, 2 of the cases had more than one tumour nodule (Rubin et al., 1958). In another series of 75 cases of pulmonary sclerosing haemangioma, 4 had multiple tumour nodules and in 2 of these the nodules were innumerable (J. T. Gmelich and A. A. Liebow, 1977, personal communication). This was a similar feature in the present case.

The pathogenesis of sclerosing haemangioma of the lung is still controversial. Rubin et al. (1958) suggest that the lesion represents a congenital weakness of blood vessels. However, it is argued that if sclerosing haemangiomas were truly due to hereditary weakness of blood vessels, multiple vascular lesions and multiple organ involvement would be the rule rather than the exception. This lesion has also been identified with the postinflammatory and xanthomatous pseudo-tumours of the lung (Fisher and Beyer, 1959). Mori (1968) suggested that the tumour is essentially a haemangiomatous lesion and the presence of histiocytes in the tumour is a result of a phagocytic response to sequestrated blood.

Electron microscopic observations on this lesion are also at variance. While Hass, Yunis and Totten (1972) conclude that the primary cellular proliferation is endothelial, the results obtained by Hill and Eggleston (1972) suggest an epithelial origin of the tumour. Electron microscopic and histochemical evidence in another study suggests that the main cell component of the tumour resembles granular pneumocyte and the lipid present in the stroma also originates from the same (Kennedy, 1973).

Nair, Nair and Weisbrot (1974) stressed the histological similarity of a case to fibrous histiocytoma, under which head the dermal sclerosing haemangiomas are also being classified.

Recently, Bahadori and Liebow (1973) have asserted that the lesion is essentially a proliferation of angioblastic tissue. The epithelial and inflammatory cell proliferation are secondary reactions. It is also stressed that sclerosing haemangiomas are distinct from post-inflammatory pseudo-tumours of 
the lung. The latter have recently been classified as xanthogranuloma, plasma cell granuloma and pseudolymphoma (Cox, Chang and Mantz, 1975).

The present authors consider the lesion described in this case most likely to be a vasoformative neoplastic lesion of multicentric origin with a slow rate of growth, remaining undetected for long periods of time and having a good prognosis.

\section{Acknowledgments}

We wish to thank Dr J. T. Gmelich and Dr A. A. Liebow, Veterans Administration Hospital, Lajolla, California, for reviewing the slides and confirming the diagnosis.

\section{References}

Arean, V.M. \& Wheat Jr., M.W. (1962) Sclerosing hemangiomas of the lung - a case report and review of literature. American Review of Respiratory Diseases, 85, 261.

BAHADORI, M. \& Liebow, A.A. (1973) Plasma cell granuloma of the lung. Cancer. New York, Philadelphia, etc., 31, 191.

Cox, I.L., Chang, C.H.J. \& Mantz, F. (1975) Pseudotumor of the lung. A case report and review stressing radiographic criteria. Chest, 67, 723.

FisHeR, E.R. \& BEYER, F.D. (1959) Post inflammatory tumor (xanthoma) of the lung. Diseases of the Chest, 36, 43.
Gross, R.E. \& Wolbach, S.W. (1943) Sclerosing hemangiomas; their relationship to dermatofibroma, histiocytoma, xanthoma and to certain pigmented lesions of the skin. American Journal of Pathology, 19, 533.

Hass, J.E., Yunis, E.F. \& TotTen, E.S. (1972) Ultrastructures of a sclerosing hemangioma of the lung. Cancer. New York, Philadelphia, etc., 30, 512.

Hill, G.S. \& EgGleston, J.C. (1972) Electron microscopic study of so called pulmonary sclerosing hemangioma. Report of a case suggesting epithelial origin. Cancer. New York, Philadelphia, etc., 30, 1072.

KENNEDY, A. (1973) Sclerosing haemangioma of the lung an alternative view of its development. Journal of Clinical Pathology, 26, 792.

Lago, J.V., Pujol, J.L., Reboiras, S.D., Larrauri, J. \& SCHACKe DE Riguel, L. (1976) Fibrous histiocytoma of the lung. Thorax, 31, 475.

Liebow, A.A. \& Hubell, D.S. (1956) Sclerosing hemangioma (histiocytoma, xanthoma) of the lung. Cancer. New York, Philadelphia, etc., 9, 53.

Mori, S. (1968) Sclerosing hemangioma of the lung. Diseases of the Chest, 54, 381 .

NaIr, S., NaIR, K. \& Weisbrot, I.M. (1974) Fibrous histiocytoma of the lung (sclerosing hemangioma variant?) Chest, 65, 465.

Rubin, E.H., Rubin, M. \& Sicklick, E. (1958) Circumscribed sclerosing hemangiomas of the lung appearing as coin lesions. Cancer. New York, Philadelphia, etc., 11, 713. 